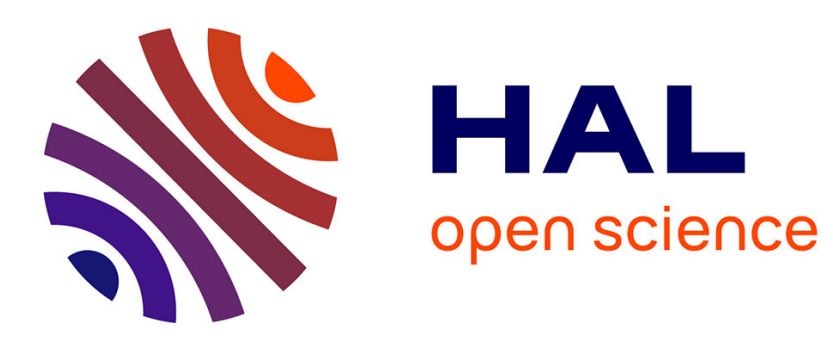

\title{
General physical studies on semiconductors using a scanning electron microscope
}

A. Gopinath, de Monts de Savasse, G.A.C. Jones

\section{To cite this version:}

A. Gopinath, de Monts de Savasse, G.A.C. Jones. General physical studies on semiconductors using a scanning electron microscope. Revue de Physique Appliquée, 1974, 9 (2), pp.347-353. 10.1051/rphysap:0197400902034700 . jpa-00243785

\section{HAL Id: jpa-00243785 https://hal.science/jpa-00243785}

Submitted on 1 Jan 1974

HAL is a multi-disciplinary open access archive for the deposit and dissemination of scientific research documents, whether they are published or not. The documents may come from teaching and research institutions in France or abroad, or from public or private research centers.
L'archive ouverte pluridisciplinaire HAL, est destinée au dépôt et à la diffusion de documents scientifiques de niveau recherche, publiés ou non, émanant des établissements d'enseignement et de recherche français ou étrangers, des laboratoires publics ou privés. 


\title{
GENERAL PHYSICAL STUDIES ON SEMICONDUCTORS USING A SCANNING ELECTRON MICROSCOPE
}

\author{
A. GOPINATH, DE MONTS DE SAVASSE and G. A. C. JONES
}

\author{
School of Electronic Engineering Science, University College of North Wales, \\ Dean Street, Bangor, Caerns., North Wales, England
}

\begin{abstract}
Résumé. - Les modes de conductibilité à faisceau induit et de cathodoluminescence ont été employés à l'étude des couches épitaxiales et des diodes d'effet Gunn. On indique dans cet exposé les techniques utilisées.

Abstract. - The beam-induced conductive-mode and cathodoluminescence mode have been used in the study of GaAs and InP epitaxial layers and Gunn devices. The paper outlines the techniques used.
\end{abstract}

1. Introduction. - The study of semiconductors and devices using the SEM has received very considerable attention to the present time. The attractions are obvious : the SEM provides a non-destructive, contactless and rapid method of examining material and devices and is ideal for uses ranging from basic studies to trouble-shooting in production lines. At present semiconductor SEM studies have exploited two specific modes of operation: the conduction mode, and the cathodoluminescence mode and the present paper discusses some aspects of each of these modes used in semiconductor and device studies in Bangor. The work in Bangor has been primarily concerned with the bulk-effect GaAs Gunn device, and therefore junction devices are not discussed.

2. Conduction mode : beam-induced conductivity. - The conduction-mode covers three variants: the specimen-current, the charge-collection, and the beam-induced conductivity modes. The specimencurrent provides little information that is not already present in the emissive signal, and is generally used for atomic number contrast with some loss of topographic information. The charge-collection mode is used where built-in junction fields are present in the specimen and is the subject of a separate paper by Bresse [1]. The beam-induced conductivity mode is capable of examining both material and devices when no built-in fields are present, provided only that the region to be examined is between two ohmic contacts. The latter mode is the only one of these three which is discussed in some detail in this section.

2.1 PHYSICAL MODEL FOR BEAM-INDUCED CONDUCTIVITY. - The electron-beam impinging on the semiconductor specimen creates hole-electron pairs and these diffuse away from the generation region as they recombine, and in the process create a region of higher conductivity. Thus, the conductivity of the material between the ohmic contacts has now been altered, and this change when monitored may be used as the video signal for the SEM. To obtain this increase in conductance a voltage is applied across the ohmic-contacts and the change in current through the specimen due to beam irradiation is detected. The voltage sets up a field in the specimen which causes a component of drift in addition to the diffusion of the hole-electron pairs, and thus modifies the beam-induced conductivity region. The magnitude of the field determines whether diffusion or drift predominates, and thus the results are different in two extreme conditions of negligible field and very high field.

In the scanning microscope the beam is scanned over the region of interest, and it is assumed that the rate of scanning is sufficiently slow so that steadystate conditions are set-up at all positions of the beam. Thus, no time-dependent effects are accounted for.

2.2 BEAM-INDUCED CONDUCTIVE MODE SIGNALS. The above physical model when analysed predicts the beam-induced signals in the SEM as a function of the beam position on the specimen and the specimen parameters : diffusion length, lifetime, mobility and applied field. The behaviour of the beam-generated hole-electron pairs is governed by the diffusion equation. In extrinsic n-type material this takes the form for the excess minority carriers (holes) :

$D_{\mathrm{p}} \nabla^{2}(\Delta p)-\mu_{\mathrm{p}} E \cdot \nabla(\Delta p)-\mu_{\mathrm{p}} p(\nabla . E)-\frac{\Delta p}{\tau_{\mathrm{p}}}=-g$

where $D_{\mathrm{p}}$ is the hole diffusion constant, $E$ is the drift field, $\mu_{\mathrm{p}}$ is the hole mobility, $\tau_{\mathrm{p}}$ is the hole- 
lifetime and $g$ the hole-electron generation rate assumed to be constant under the beam-spot, and zero elsewhere. For extrinsic material the spacecharge may be neglected on the basis of quasi-neutrality and thus the third term in the above equation may be ignored. With near intrinsic material, this term may also be neglected, in which case the diffusion and mobility constants take " effective" values [2]. If we may assume that the hole-electron distribution is uniform in the plane normal to the field, the above equation reduces to the one dimensional form. This is solved analytically or numerically with the boundary conditions that the excess hole-electron concentration is zero at the contacts.

With this excess distribution known, it now remains for the change in conductivity to be estimated. To obtain this we assume for a rectangular sample, that only a narrow longitudinal strip of the sample, two diffusion lengths $\left(2 L_{\mathrm{p}}\right)$ wide, and one diffusion length $\left(L_{\mathrm{p}}\right)$ deep is affected, and the distribution of the hole-electron pairs in this is known (see Fig. 1).

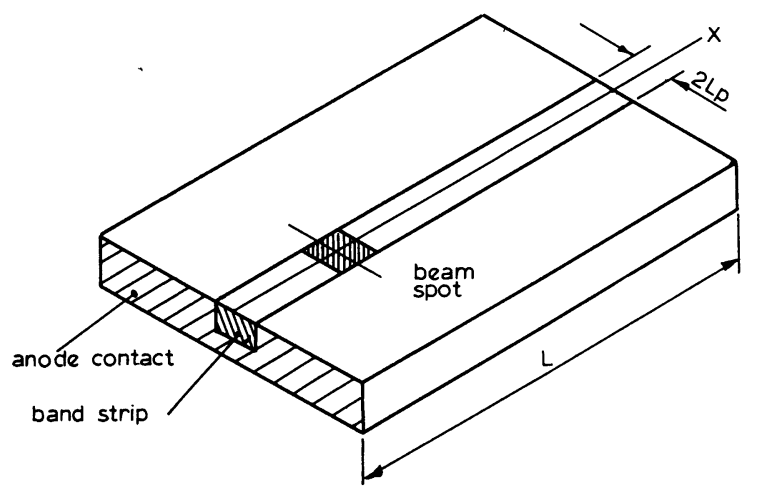

(a)

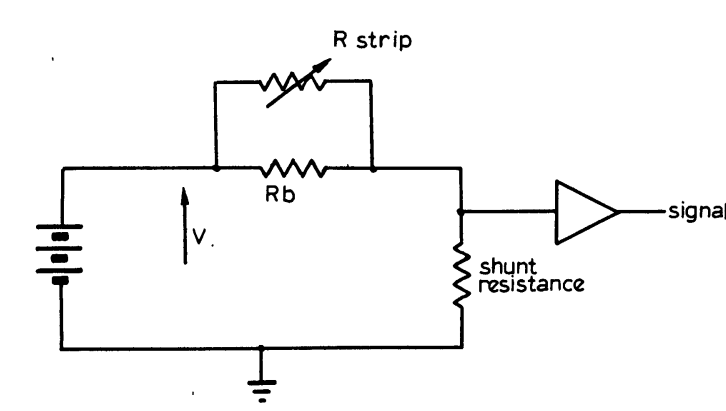

(b)

FIG. 1. - Schematic diagram of irradiated strip in rectangular geometry sample, together with equivalent circuit.

For a constant voltage excitation it can be shown that the change in current due to beam irradiation for n-type extrinsic materials is given by :

$$
\Delta I=\frac{V q(1+b) \mu_{\mathrm{p}}}{R^{2} A_{0}} \int_{0}^{L} \frac{\Delta p(x)}{\sigma_{0}^{2}} \mathrm{~d} x
$$

where $V$ is the applied voltage, $q$ is the electronic charge of each hole, $b$ is the ratio of electron to hole mobility, $R$ is the unperturbed resistance of the strip, $\sigma_{0}$ the unperturbed specimen conductivity, $A_{0}$ the strip sectional area and $L$ is the device length. With $\Delta p(x)$ known from the diffusion equation, it follows that $\Delta I$ can be estimated for different beam positions, for different magnitudes of applied field (normalised to the diffusion field). Curves of the distribution of $\Delta p$ for the specific case of the diffusion length given by $L_{\mathrm{p}}=0.1 \mathrm{~L}$, is shown for two beam positions in figure 2, for different applied fields. These distributions are valid only if $\Delta p \ll n_{0}$, since the unperturbed field is used in the diffusion equation solution. The variation of signal (which is proportional to $\left.\int_{0}^{L} \Delta p \mathrm{~d} x\right)$ with beam position is shown in figure 3 for three different values of diffusion to device length for a device of uniform conductivity. From these we note that at low fields the signal is flat over the whole device, and at high fields becomes triangular, peaking at the anode for n-type material. The distribution curves in figure 2 indicate that the reason for this variation is the effect of drift and the boundary conditions on the carriers, and it follows that if good spatial resolution is required in this mode then the applied field must be smaller than the diffusion field.

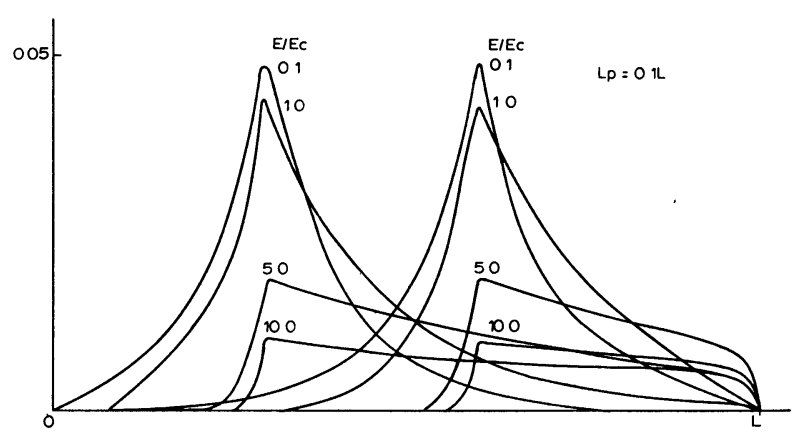

FIG. 2. - Predicted one dimensional distribution of excess minority carriers for a rectangular geometry sample length $L$, with $L_{\mathrm{p}}=0.1 L$ for three different beam positions and various applied fields (normalised to the diffusion field).

For the annular geometry device, the strip model is modified by a pseudo two-dimensional approach [3]. Computed curves of the video signal $\Delta I$ are shown in figure 4 for $L_{\mathrm{p}}=0.01 a_{0}$, where $a_{0}$ is the outer radius, and $a_{\mathrm{i}}=0.33 a_{0}$, where $a_{\mathrm{i}}$ is the interna radius of the annulus, and shows a geometric contrast effect. Supporting experimental results for both these predictions have been published elsewhere [3].

From the curves in figures 2 and 3 it is possible to estimate the magnitude of diffusion field and diffusion length, by examining the change of video signal with applied bias. This provides an approximate method, which is correct to within an order of magnitude. 


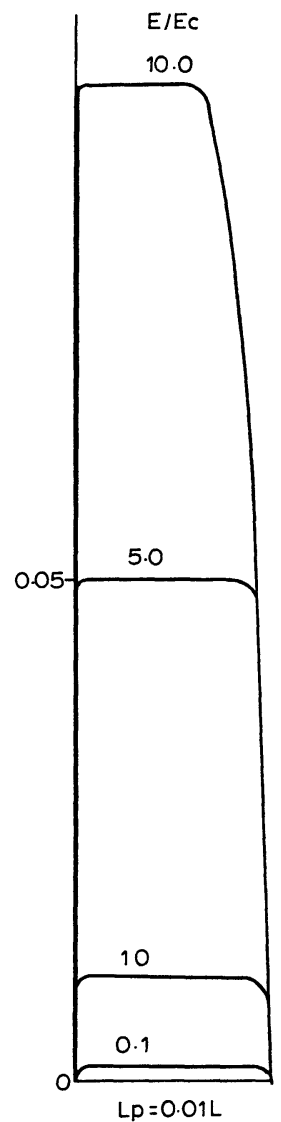

(a)

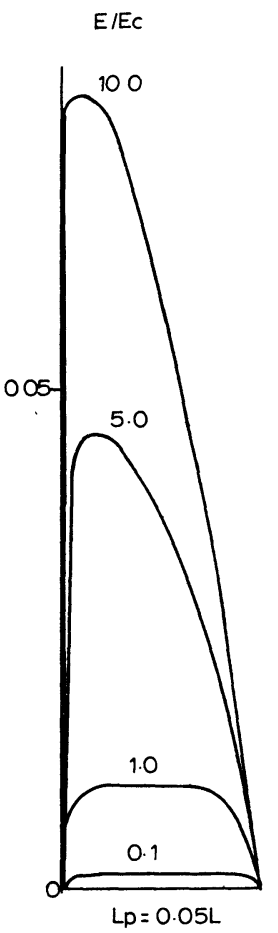

(b)

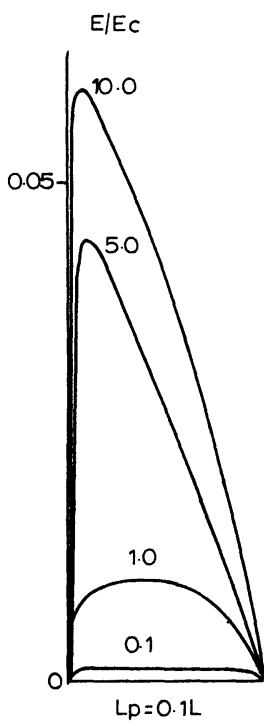

(c)
FIG. 3. - Predicted variation of conductive mode signal with beam position in uniform conductivity rectangular geometry samples of different diffusion lengths with various applied fields.

In the above discussion the effect of surface recombination velocity has been neglected and indeed the one-dimensional model does not allow for these effects. Munakata [4] has recently carried out work in which he shows that surface recombination velocity is important under certain conditions only. However, his results are too complex to produce universal curves of the form shown in figures 2 and 3 . If surface recombination velocity is high, estimated diffusion lengths from the curves in these figures will be in error, and generally lower than bulk values.

\subsection{INHOMOGENEITIES IN BEAM-INDUCED CONDUCTIVE} MODE. - From eq. (2) it can be seen that contrast is also a function of $1 / \sigma^{2}$, and thus small resistivity variations should show up in this mode. The resolution however, is dependent on the applied field, which should be much smaller than the diffusion field for resistivity studies. Thus, increasing the bias should smear out these variations considerably.

Inhomogeneities which are regions of different generation or recombination rates, will show up under most bias conditions. Under the beam spot, the change in $g$ or $\tau$ will alter the magnitude of carriers flowing outward, and thus modify the signal linearly.

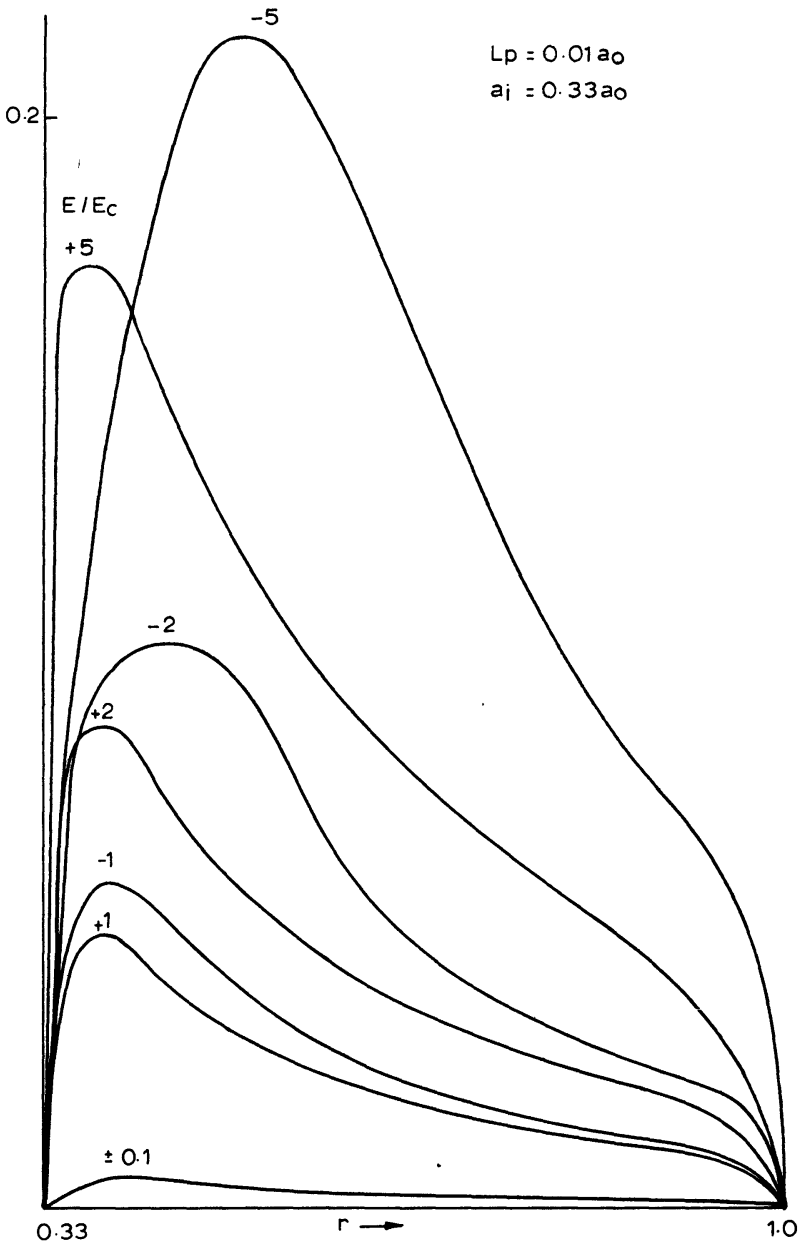

FIG. 4. - Predicted variation of conductive mode signal with beam position in a uniform conductivity annular geometry sample, diffusion length $L_{\mathrm{p}}=0.01 a_{0}$, inner radius $a_{\mathrm{i}}=0.33 a_{0}$, outer radius $a_{0}$ normalised to unity. Applied field normalised to diffusion field at outer radius.

Outside the beam-spot region, high recombination regions will also show up, though less markedly, when they are in beam-induced conductivity regions.

Figure 5 shows a set of micrographs where these affects have been noted. Figure $5, a$ ) and $b$ ) show micrographs of inhomogeneities in $2 \mu \mathrm{m}$ epitaxial layers of $\mathrm{n}$-type $\mathrm{GaAs} \sim 10^{16}$ electrons grown on a semi-insulating substrate, taken with a $5 \mathrm{kV}$ beam. The same device as in $5, b$ ) was examined at much higher applied field in $c$ ) and reversed field $d$ ) : note that contrast here is as predicted in figure 2.

Other studies on similar layers show the effect of beam penetration. The two micrographs of a device (similar to that in $5, b$ )) shown in figure 6 were taken under low-field conditions with beam voltages at $3.5 \mathrm{kV}$ and $10 \mathrm{kV}$ respectively, where the penetration range is approximately $0.2 \mu \mathrm{m}$ and $0.9 \mu \mathrm{m}$ respectively when calculated by the Archard model [3]. Note the large number of black spots in $6, b$ ) indicating that these are probably inhomogeneities at the interface of the substrate and the epitaxial layer. The low beam-voltage micrograph should also show up some 

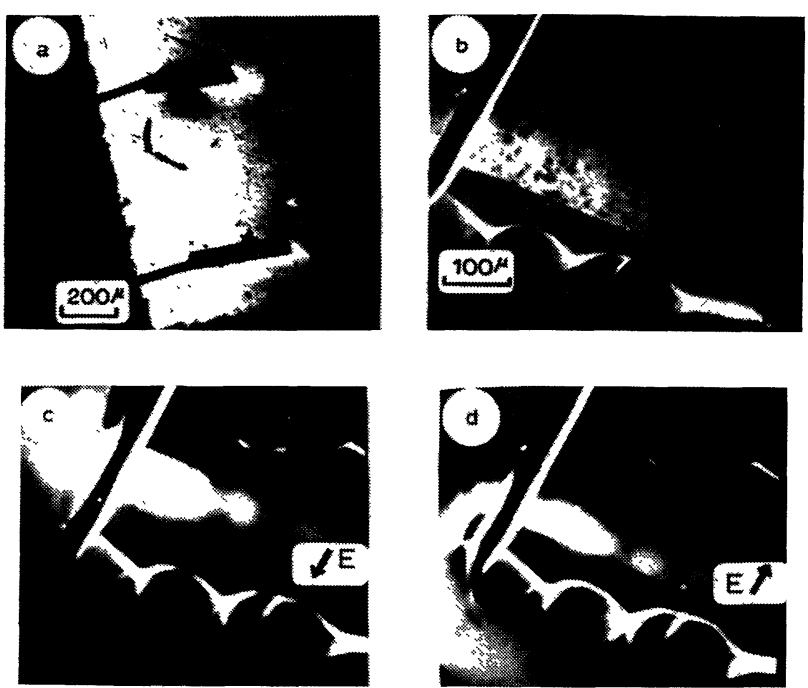

FIG. 5. $-a), b$ ) Inhomogeneities in a $2 \mu \mathrm{m}$ epitaxial layer of n-type GaAs, $10^{16}$ electrons per cc, grown on semi-insulating substrate. Beam voltage $5 \mathrm{kV}$. Bias fields $E<E_{\mathrm{c}}$ diffusion field. $\left.E=20 \mathrm{~V} / \mathrm{cm}, E_{\mathrm{c}}=130 \mathrm{~V} / \mathrm{cm} ; c\right), d$ ) Same sample as in $b)$ above $E>E_{\mathrm{c}}$. Note that contrast is as predicted in figure 2 for this condition. $E=900 \mathrm{~V} / \mathrm{cm}, E_{\mathrm{c}}=130 \mathrm{~V} / \mathrm{cm}$.

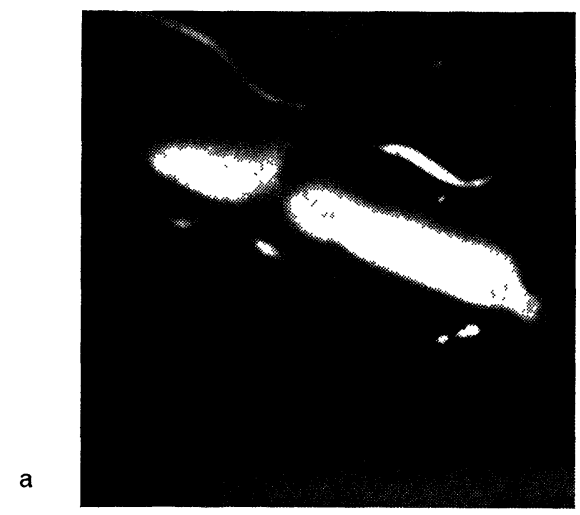

3-5 kV

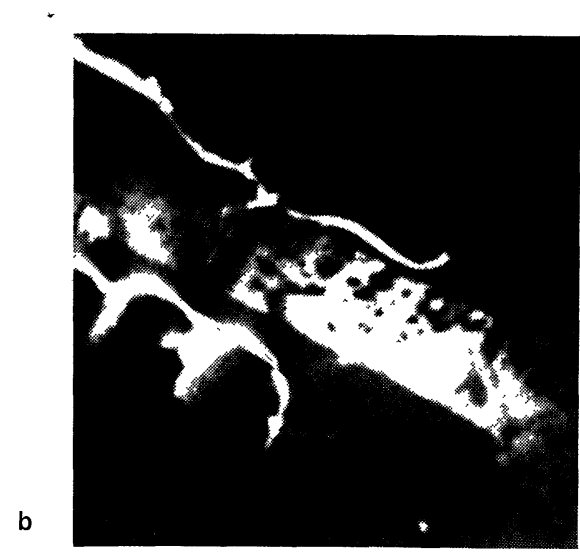

$10 \mathrm{kV}$

FIG. 6. - $-a$ ) Device similar to that in figure $5 b$ examined with $E<E_{\mathrm{c}}$, examined with $3.5 \mathrm{kV}$ beam. $b$ ) Device as in $6 a$ examined with $10 \mathrm{kV}$ beam, same field, $E<E_{\mathrm{c}}$. of these spots as the diffusion length is about $2 \mu \mathrm{m}$ in this material but surface recombination is probably the dominant mechanism here and hence only a few emerge.

In summary, we have shown that it is possible to predict beam-induced conductive mode signals in semiconductors and devices using a simple model, provided that the region of interest is between two ohmic contacts. It is also possible to make approximate estimates of diffusion and lifetime with this mode. Resistivity variations and inhomogeneities may also be examined with this technique.

3. Cathodoluminescence mode. - The cathodoluminescence (CL) mode may only be used with direct-gap semiconductors, where the recombination of the beam-generated hole-electron pairs is radiative, i. e. results in the emission of photons. Typical material studied in this mode are GaAs, GaP and a large variety of "phosphors " used in cathode-ray tubes. The work at Bangor has examined the temperature dependence of luminescence in GaAs, n-type with $\sim 10^{16}$ electrons/cc concentration. The luminescence mode has been used in estimating the uniformity of epitaxial layers of InP, and has also been used as a method of estimating temperature distributions in GaAs Gunn devices. In this section we discuss each of these aspects in some detail.

3.1 Temperature DePendence OF CL IN GaAs. The temperature dependence of $\mathrm{CL}$ in direct gap semiconductors may be obtained using the Roosbroeck-Shockley [5] equation which gives the total output $P$ in terms of the excess minority carrier concentration profile $\Delta p(z)$ (for n-type material) and the absorption coefficient $\alpha(v)$ :

$$
\begin{aligned}
P=\frac{8 \pi n^{\prime}}{c^{2}}\left(\frac{A^{\prime}}{A}\right. & \left.\frac{1}{n_{0}}+\frac{1}{p_{0}}\right) \times \\
& \times \int_{v=0}^{\infty} \alpha(v) v^{2} \exp \left(\frac{-h v}{K T}\right) \eta(v) \mathrm{d} v \\
& \times \int_{n=0}^{\infty} \Delta p(z) \exp (-\alpha(v) z) \mathrm{d} z
\end{aligned}
$$

where $n^{\prime}$ the material refractive index, $c$ is the velocity of light, $\left(A^{\prime} / A\right)$ is the degeneracy factor, $n_{0}, p_{0}$ are the equilibrium electron, hole concentration, $v$ is the frequency of emitted light, $\Delta p(z)$ is the minority carrier concentration profile, and $\eta(v)$ is the frequencydependent detector sensitivity.

In principle the $C L$ variation with temperature may be estimated if the temperature dependence of each of the above terms is known. In practice a series of assumptions have to be made to evaluate this dependence and simplify the computation. The exponential term $\exp (-\alpha z)$ may be omitted as the limits of $z$ in the integral for SEM beam voltages of 5 to $20 \mathrm{kV}$ is small. The excess minority profile 
can be obtained from diffusion eq. (1), with no field present. The geometry in this case, however, is somewhat different; the semiconductor slab surface is assumed to be normal to the beam ( $z$-directed) and the slab is assumed to extend a large distance in the $z$-direction. The diffusion equation is then solved in the $z$-dependent one-dimensional form for a generation profile given by Everhart and Hoff [6], with the boundary conditions set for the appropriate recombination velocity at the surface and $\Delta p(\infty)=0$. With the temperature dependence of the lifetime and diffusion-length known, the temperature variation of the integral $\int \Delta p(z) \mathrm{d} z$ is obtained. When the temperature dependence of $\alpha(v)$ and $n_{0}, p_{0}$ are included in eq. (3), the variation of CL output $P$ with temperature can be estimated. Results of this calculation compared with experiment is shown in figure 7 for

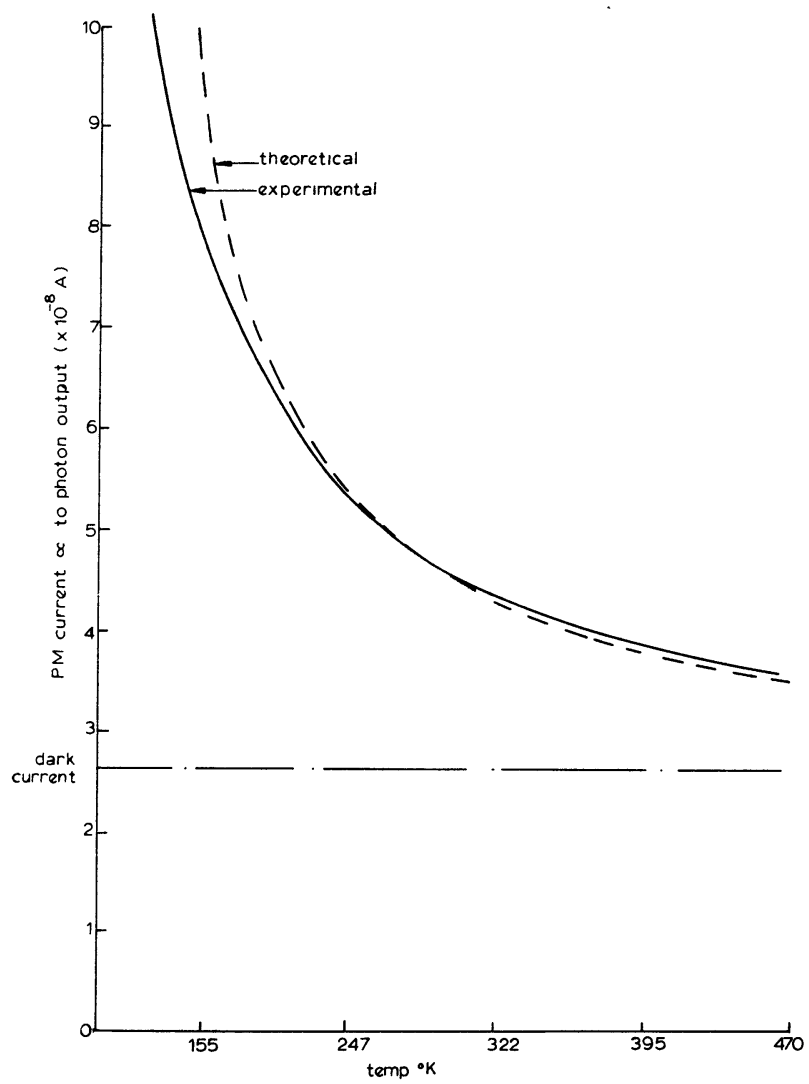

Fig. 7. - Curves showing theoretical and experimental results of temperature dependence of cathodoluminescence output from GaAs, n-type, $2 \times 10^{16}$ electrons per cc. The theoretical curve was normalised to coincide with experimental results at room temperature. The sample was examined with a $15 \mathrm{kV}$ beam, $1.42 \times 10^{-7}$ A current.

n-type GaAs $2 \times 10^{16}$ electrons/cc in the temperature range $150 \mathrm{~K}$ to $500 \mathrm{~K}$, with a $15 \mathrm{kV}$ beam. Details of this calculation and experiment are to be published elsewhere [7].
3.2 Doping Profiles. - From eq. (3) the doping dependence arises from the term $\left(\frac{A^{\prime}}{A} \frac{1}{n_{0}}+\frac{1}{p_{0}}\right)$. For non-degenerate direct-gap semiconductors $A^{\prime} \mid A=1$ and $p_{0}=n_{\mathbf{i}}^{2} / n_{0}$, and for extrinsic $« n »$ material $p_{0} \ll n_{0}$.

Thus

$$
\left(\frac{A^{\prime}}{A} \frac{1}{n_{0}}+\frac{1}{p_{0}}\right) \simeq \frac{n_{0}}{n_{\mathrm{i}}^{2}}
$$

and the luminescence output is proportional to doping concentration.

Figure $8 a$ shows a CL micrograph $(\times 410)$ of a longitudinal InP Gunn device mounted on a conical metallic header, and the bright region is the $n^{+}$ substrate. At higher magnification $(\times 13500)$ at the top contact edge in $8 b$ the active $" n$ » layer can be seen. CL lines can taken across this " $n$ " layer show its uniformity, which is in agreement with other doping profile measurements [8].

3.3 Temperature Profiles In GaAs GunN DEVICES. - From the temperature dependence studies in Section 3.1, it follows that the temperature distribution in an operating GaAs or other such devices can be obtained provided the luminescence intensity with temperature is calibrated. All GaAs Gunn devices operate with very high fields and therefore any beam generated carriers will drift, and the spatial resolution of the temperature distribution will be degraded. Thus, the study of temperature profiles would require the device bias to be pulsed and the luminescence sampled during the off period only. The CL output however is extremely sensitive to small doping fluctuations and therefore it becomes necessary to calibrate the luminescence intensity in the region of particular interest. For this, the device is mounted on a hot stage, and under no bias conditions, CL calibration linescans are taken at various known temperatures with a particular sampling pulse. Next, the device is operated under normal pulsed conditions and the luminescence intensity sampled over the same region with the same sample pulse. Superposition of this on the calibration curves gives the quantitative temperature profile required. The estimated spatial resolution is about $2 \mu \mathrm{m}$, and temperature resolution is about $\pm 4 \mathrm{~K}$.

Figure $9 b$ shows a set of calibration curves taken on a transverse Gunn device sketched schematically in $9 a$. The operating CL profiles are shown in $9 c$, and superposition of these on $9 b$ gives the temperature profiles in this type of device. Other studies on device-chip time-constant are also possible by this technique [9].

In summary, the luminescence output is given by the Roosbroeck-Shockley equation. The temperature dependence of $C L$ has been estimated using this equation and compared with experiment. The lumi- 


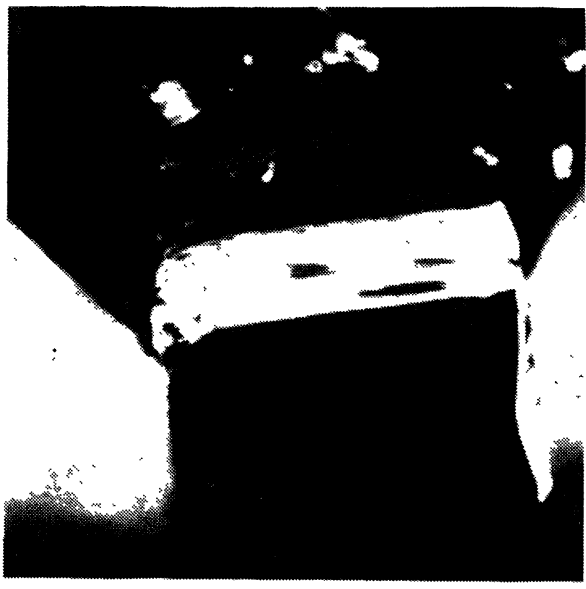

(a)

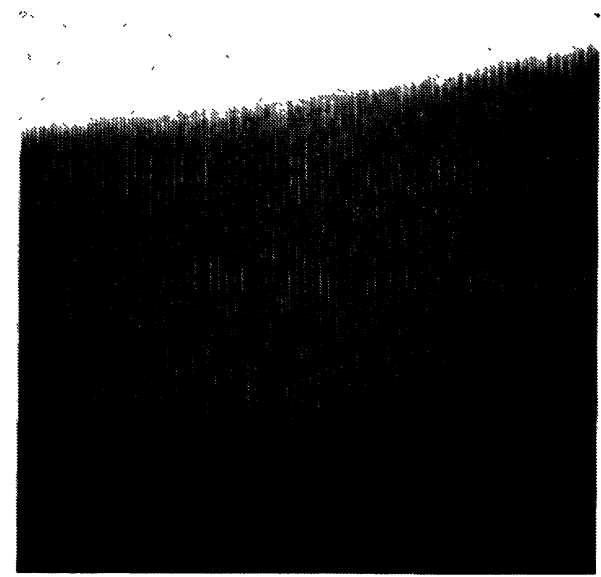

$\times 13,500$

(b)

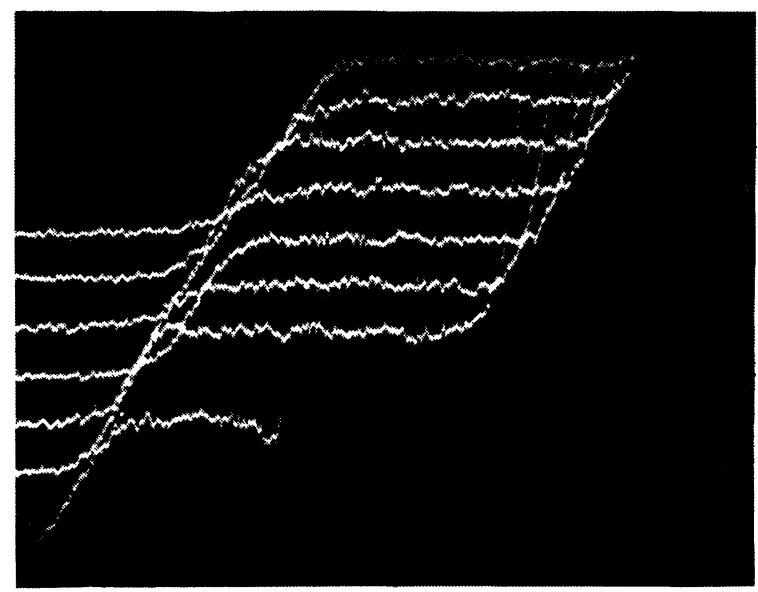

(c)

FIG. 8. - a) Cathodoluminescence micrograph of a longitudinal InP Gunn device mounted on a conical metallic header $\times 410 . b)$ Same device at higher magnification near the top metal contact, showing the n-layer, $12 \mu \mathrm{m}$ wide $\times 13500$. c) CL linescan across n-layer showing doping uniformity.

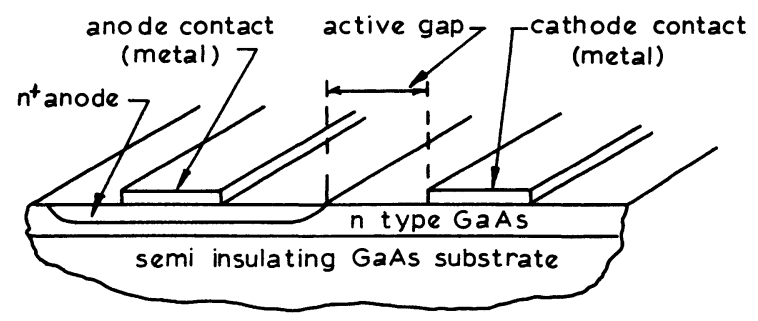

(a)

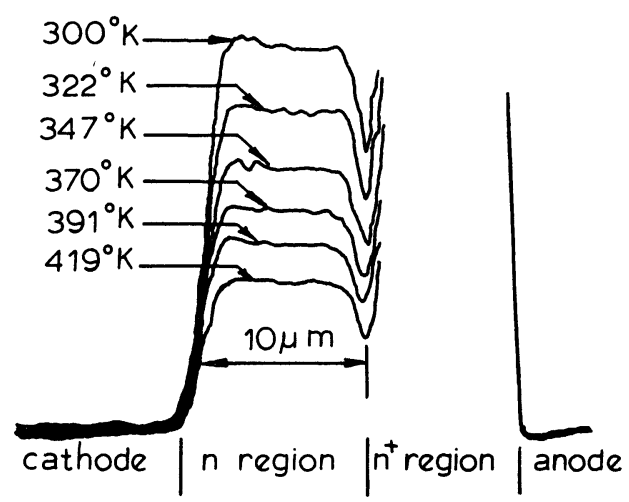

(b)

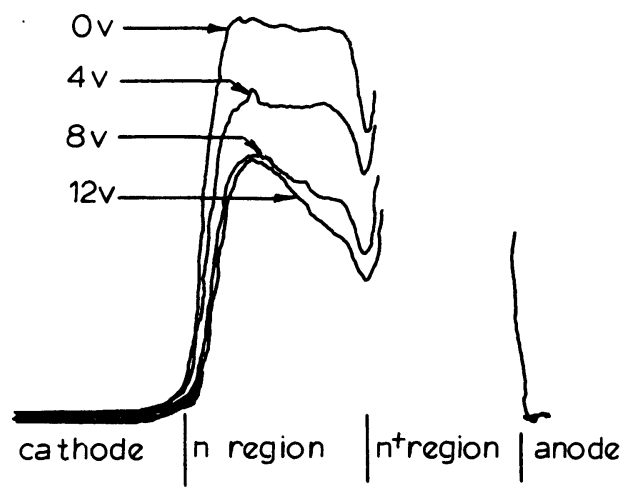

(c)

FIG. 9. - a) Schematic diagram of a GaAs transverse Gunn device showing structure of active layer. $b$ ) Calibration CL linescans across device held at constant temperature. $c$ ) Linescans across device under different bias conditions. Threshold at $7 \mathrm{~V}$.

nescence intensity is a linear function of doping in non-degenerate extrinsic material and hence can be used to estimate the uniformity of doping profiles. The CL variation has also been used to estimate temperature profiles in GaAs Gunn devices with a spatial resolution of better than $2 \mu \mathrm{m}$ and temperature resolution of $\pm 4 \mathrm{~K}$.

4. Conclusions. - This paper has outlined techniques for the study of semiconductors and devices in two modes (beam-induced conductive and cathodoluminescence modes) of the SEM. The basic physical model in each mode has been analysed and the 
predicted signals have compared favourably with experiment. Applications of these modes to the study of specific aspects of semiconductors and devices have been outlined.
Acknowledgments. - My thanks are due to my students M. T. de Monts de Savasse and Mr, G. A. C. Jones, without whom this work would not have been performed at Bangor.

\section{References}

[1] Bresse, J. F., Revue Phys. Appl. 9 (1974) 354.

[2] Sмітн, R. A., Semiconductors (Cambridge University Press) 1957.

[3] Gopinath, A., de Monts de Savasse, T., J. Phys. D (Applied Physics) 4 (1971) 2031-2038.

[4] Munakata, C., Japan J. of Appl. Phys. 11 (1972) 869-873.

[5] van Roosbroeck, W., Shockley, W., Phys. Rev. 94 (1954) 1558-1560.
[6] Everhart, T. E., Hoff, P., J. Appl. Phys. 42 (1971) $5837-$ 5846.

[7] Jones, G. A. C., NAG, B. R., Gopinath, A., to be published in J. Phys. D (Applied Physics) (1974).

[8] Colliver, D. J., private communication.

[9] Jones, G. A. C., Gopinath, A., Proc. of the scanning electron microscopy conference at Newcastle, July 1973, to be published by Institute of Physics, UK. 